\title{
New investigations around CYP11A1 and its possible involvement in an androstenone QTL characterised in Large White pigs
}

\author{
Annie Robic ${ }^{1 *}$, Guillaume Le Mignon², Katia Fève ${ }^{1}$, Catherine Larzul $^{2}$ and Juliette Riquet ${ }^{1}$
}

\begin{abstract}
Background: Previously, in boars with extreme androstenone levels, differential expression of the CYP11A1 gene in the testes has been characterised. CYP11A1 is located in a region where a QTL influencing boar fat androstenone levels has been detected in a Large White pig population. Clarifying the role of CYP11A1 in boar taint is important because it catalyses the initial step of androstenone synthesis and also of steroid synthesis.

Results: A genome-wide association study located CYP11A1 at approximately $1300 \mathrm{~kb}$ upstream from SNP H3GA0021967, defining the centre of the region containing the QTL for androstenone variation. In this study, we partially sequenced the CYP11A1 gene and identified several new single nucleotide polymorphisms (SNP) within it. Characterisation of one animal, heterozygous for CYP11A1 testicular expression but homozygous for a haplotype of a large region containing CYP11A1, revealed that variation of CYP11A1 expression is probably regulated by a mutation located downstream from the SNP H3GA0021967. We analysed CYP11A1 expression in LW families according to haplotypes of the QTL region's centre. Effects of haplotypes on CYP11A1 expression and on androstenone accumulation were not concordant.

Conclusion: This study shows that testicular expression of CYP11A1 is not solely responsible for the QTL influencing boar fat androstenone levels. As a conclusion, we propose to refute the hypothesis that a single mutation located near the centre of the QTL region could control androstenone accumulation in fat by regulating the CYP11A1 expression.
\end{abstract}

\section{Background}

Boar taint refers to an unpleasant odour and flavour of meat which occurs in a high proportion of uncastrated male pigs and is primarily due to the accumulation of androstenone and skatole in fat tissue [1,2]. Androstenone is synthesised in the testis, together with the steroid hormones, androgens and estrogens, from pregnenolone [3-5], in relation to sexual development and is stored in fat tissue because of its lipophilic properties.

Currently, only a few studies have tried to identify QTL for androstenone accumulation [6-9]. It is important to understand the genetic mechanisms controlling this trait in order to be able to select pigs for low androstenone levels and thus limit the occurrence of boar taint.

\footnotetext{
* Correspondence: annie.robic@toulouse.inra.fr

'INRA, UMR444, Laboratoire de Génétique Cellulaire, 31326 Castanet-Tolosan, France

Full list of author information is available at the end of the article
}

Le Mignon et al. [10] identified QTL for androstenone variation in a 480 Large White (LW) pig population using the Illumina PorcineSNP60 BeadChip. The present study focused on one of these QTL, explaining $18.7 \%$ of the genetic variance, which was detected on the q-arm of chromosome Sus scrofa 7 (SSC7) using GWAS (Genome Wide Association Studies) near the position $66 \mathrm{Mb}$ on the "Sscrofa9" version (April 2009) of the pig genome sequence. Examination of the gene content in this QTL region, suggested CYP11A1 as an obvious candidate gene. Moe et al. [11] and Grindfleck et al. [12] had already detected differential expression of CYP11A1 in the testes of boars with either extremely high and or low levels of androstenone in fat. Moreover, a previous study reported one polymorphism in exon 1 of CYP11A1 significantly associated with androstenone levels in Yorkshire boars [13]. This gene encodes the CYP11A1 enzyme, which is localized in the mitochondrial inner membrane, and
Ciomed Central

() 2011 Robic et al; licensee BioMed Central Ltd. This is an Open Access article distributed under the terms of the Creative Commons Attribution License (http://creativecommons.org/licenses/by/2.0), which permits unrestricted use, distribution, and reproduction in any medium, provided the original work is properly cited. 
catalyses the conversion of cholesterol to pregnenolone in the first and rate-limiting step of the synthesis of steroid hormones [14]. Therefore, it is very important to clarify the role of $C Y P 11 A 1$ in boar taint. If testicular expression of CYP11A1 is found to influence the QTL for androstenone variation, it would be difficult to select against this QTL without encountering reproduction problems.

\section{Methods}

\section{Animals and samples}

On the INRA experimental farm, 98 LW sows were inseminated with 56 LW boars, chosen as unrelated as possible. Each boar inseminated one or two sows. A total of 580 male piglets were raised in pens till they reached $110 \mathrm{~kg}$ of live body weight and then slaughtered in a commercial slaughterhouse. A total of 480 animals were measured for backfat androstenone levels.

Six litters were produced by inseminating LW sows with semen from two commercial LW boars. Twentytwo animals were produced and then slaughtered at 2425 weeks of age. Testicular samples were collected immediately after slaughter, frozen in liquid nitrogen and stored at $-80^{\circ} \mathrm{C}$. To obtain testicular samples, testes were decapsulated to remove connective tissues, fasciae and the main blood vessels. Samples $\left(2\right.$ to $\left.5 \mathrm{~cm}^{3}\right)$ were collected from the inner part of the testicular tissue, containing Leydig cells.

\section{Real time PCR}

Samples were disrupted, homogenised and ground to a fine powder by rapid agitation for $1 \mathrm{~min}$ in a liquidnitrogen-cooled grinder with stainless steel beads before RNA extraction. Total RNA was isolated from testis using Total Quick RNA (Talent) kits according to the manufacturers' instructions, and treated with DNase to remove contaminating DNA. RNA concentration was determined using the NanoDrop ND-1000 spectrophotometer (NanoDrop Technologies, DE, USA). First strand cDNA synthesis was conducted using SuperScript ${ }^{\mathrm{TM}}$-II Rnase H- Reverse Transcriptase (Invitrogen, Carlsbad, CA). According to the manufacturer's instructions, $0.5 \mu \mathrm{g}$ of total RNA from each sample was used as a template with dN9 random primers (Ozyme, New England Biolabs), in a total volume of $100 \mu \mathrm{L}$.

The level of CYP11A1expression was determined by real-time PCR on cDNA from testes. Experiments were performed on the ABI 7900HT (Sequence Detection System $7900 \mathrm{HT}$ ) in a 384-well plate. All measurements were performed in duplicate on the same plate and no reference sample was used. Primers were designed in exon 3 (TGTTTCGCTTCGCCTTTGA) and exon 4 (CCCAGGCGCTCTCCAAAT) of CYP11A1 cDNA. Transcript's concentrations were corrected with respect to the housekeeping gene, TOB2B (GGGATGTCTGAAGAAGTACGAAAC//CATTCCTACAAGCCATTCCTTACG). Data was analysed with ABI software to obtain $\mathrm{Ct}$ values (threshold cycle). Four points of dilutions of a mix of cDNA were used for each gene and for each tissue, to determine PCR efficiency (E). As efficiency levels were similar for all measured genes (including the reference gene), results are expressed as $\mathrm{E}^{\left(\mathrm{Ct} \_ \text {ref }-\mathrm{Ct}_{-} \text {gene }\right)} \times$ 1000 in arbitrary units.

Before quantifying $C Y P 11 A 1$ transcripts, possible alternative transcripts were compiled from databases and we checked that the alternative transcript (AK235955 or DB787788) with a 3' shortcut exon 3 was absent in the testicular cDNA.

\section{Sequences}

Sequencing of BAC CH242-402H17 containing CYP11A1 gene related sequences is underway and subclone sequences are being captured by a blast procedure (http://www.ncbi.nlm.nih.gov/genome/seq/BlastGen/ BlastGen.cgi?taxid=9823) from the "traces-other" database.

To find polymorphisms, PCR products were produced with genomic DNA from several animals. To sequence the PCR products, an aliquot ( 1 to $12 \mu \mathrm{L})$ was purified by a single treatment $\left(45 \mathrm{~min}\right.$ at $37^{\circ} \mathrm{C}$ followed by $30 \mathrm{~min}$ at $80^{\circ} \mathrm{C}$ ) using $0.5 \mathrm{U}$ of Shrimp alkaline phosphatase (Promega) and $0.8 \mathrm{U}$ of exonuclease I (New England Biolabs). Sequencing was done with a 3730 ABI capillary DNA sequencer using a Big Dye terminator V3.1 cycle sequencing kit.

\section{Statistical analysis}

Differences between two groups of animals were assessed with a heteroscedastic Student's t-test as proposed by MS-Excel (Microsoft Corporation).

\section{Results and discussion \\ QTL region}

The 18 informative SNP present in the region containing the QTL for androstenone variation were classified into four groups based on their positions in Mb (Figure 1). The position of this QTL was arbitrarily defined in a window of $3 \mathrm{Mb}$ around the most highly associated marker, H3GA0021967 (named M11 and located at 65.91 Mb on the "Sscrofa9" provisional genome sequence), identified by GWAS analysis [10]. Since sequencing of the porcine genome is not yet completed [15], our results are anchored on the human map. The genomic content of this region $(64.4-67.4 \mathrm{Mb})$ was deduced from the global alignment proposed by the Narcisse software [16]. The first part of the QTL region between 64.40 and $66.45 \mathrm{Mb}$ corresponded to the CYP11A1-GRAMD2 region from HSA15 (Figure 1) and the second part between 66.45 and 


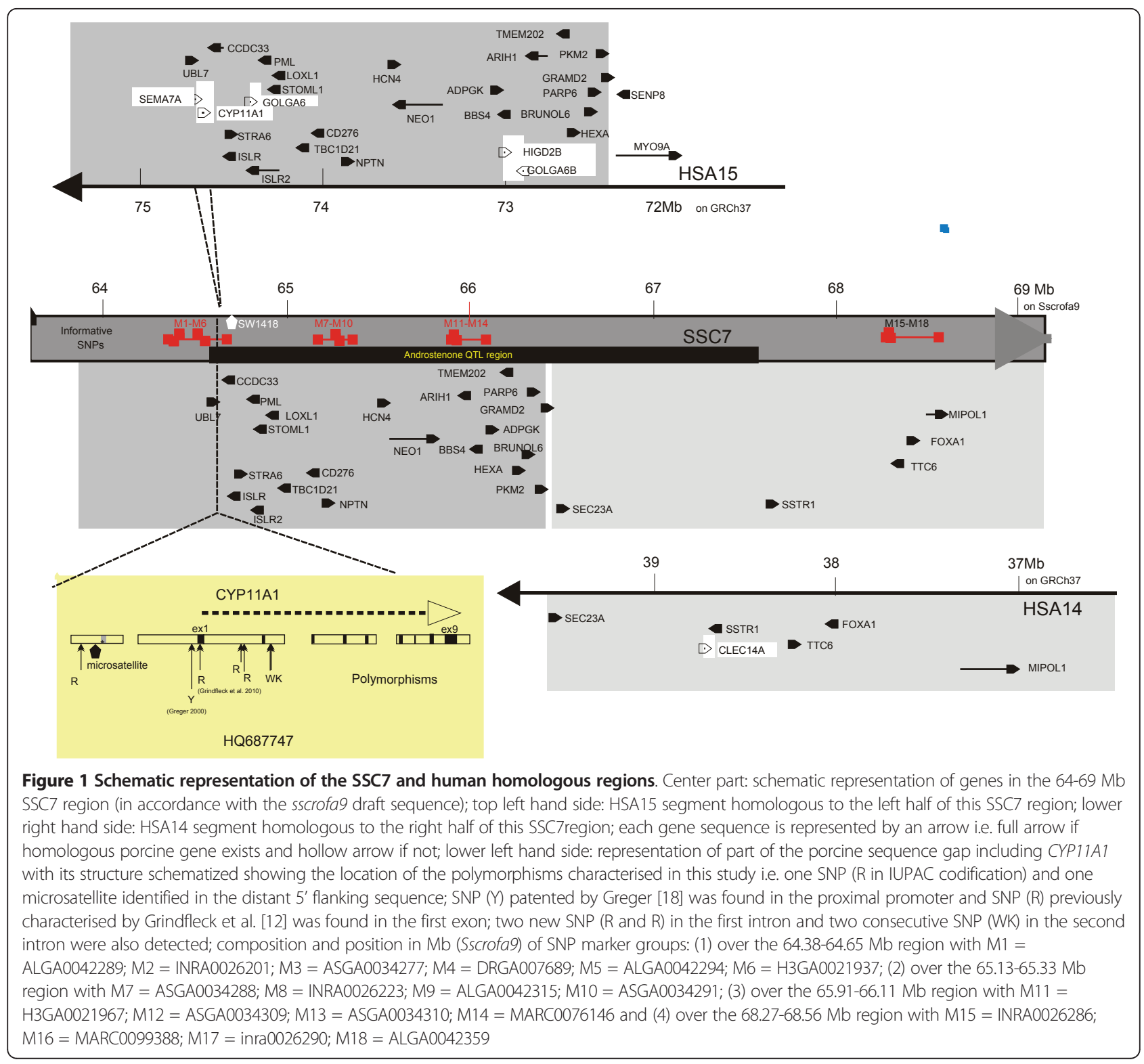

67.40 Mb was homologous to HSA14 (SEC23A-SSTR1). The information was completed and compared to the provisional annotation of the "Sscrofa9"version available on the Ensembl web site (http://www.ensembl.org/). We found that the assembly between 66.0 and $66.4 \mathrm{Mb}$ did not coincide completely although the SNP order was correct (Figure 1). Moreover, four small gaps were detected (Figure 1) and in particular, close to the porcine position 64.6 Mb. Using the Blast procedure available on the Ensembl web site, we showed that the $55 \mathrm{~kb}$ fragment on "Sscrofa9" separating the extremities of UBL7 and $C C D C 33$ genes did not contain sequences related to the SEMA7A and CYP11A1 genes. Curiously, on the human sequence, this region extended over a total of about
$73 \mathrm{~kb}$ and contained these two genes which did not overlap but extended over 25 and $30 \mathrm{~kb}$, respectively.

\section{Porcine CYP11A1 gene}

CYP11A1 appeared to be a promising candidate gene for the QTL for androstenone variation since its product, the CYP11A1 enzyme, catalyses the initial step of androstenone synthesis. Moreover, Moe et al. [11] and Grindfleck et al. [12] had already detected differential expression of CYP11A1 in the testes of boars with various extreme androstenone levels in fat. The cDNA of CYP11A1 is known in pig (NM_214427) and the sequencing of BAC $\mathrm{CH} 242-402 \mathrm{H} 17$ is underway. We performed several assemblies of sub-clone sequences 
starting with exon sequences. To capture the 5' flanking sequence, we used human sequences and in particular a regulatory region (HSA15:74665162-74667983). We were able to propose an initial assembly (HQ687747) with four genomic fragments schematized on Figure 1. CYP11A1 gene is composed of nine exons in most mammals. Pig intron 1 (3205 bp) is significantly shorter than the human counterpart (19261 bp) but longer than the mouse one (975 bp). To characterise polymorphisms, the corresponding human $19 \mathrm{~kb}$ long intron 1 was sequenced in several animals chosen from the $480 \mathrm{LW}$ animals. One SNP ( $\mathrm{R}$ according to IUPAC codification) and one microsatellite were identified in the distant 5' flanking sequence. SNP (Y) patented by Greger [17] was found in the proximal promoter and SNP (R), previously characterised by Grindfleck et al. [12], was found in the first exon. In the first intron, two new SNP ( $R$ and $R$ ) and in the second intron, two consecutive SNPs (WK) were detected. Thus, seven SNP and two microsatellites (with SW1418) were available to explore the CYP11A1 region.

\section{Expression of CYP11A1}

It would have been interesting to analyse variations of the testicular CYP11A1 expression in animals from this LW population but no samples were available. Fortunately, expression of CYP11A1 in the testis of animals from other LW families could be estimated by real-time PCR. The results are shown in Table 1 . In family A, the level of CYP11A1 expression ranged between 200 and 600 A.U. in the seven animals analysed, while in family $B$, two animals with a very high level of CYP11A1 expression (1425) and two animals with a low level (250) were found. Moreover four microsatellites were genotyped around CYP11A1 [see additional file 1, Table S1], which allowed to deduce that the boar is homozygous -/-, the sow (61043) of family A is homozygous -/- and the sow (65472) of family B is heterozygous +/- for CYP11A1expression level. Nevertheless, it is very likely that this latter sow (65472) is homozygous for the entire haplotype over this region [see additional file 1 , Table S1].

\section{Haplotype analysis in the CYP11A1 region}

Sow 65472 was genotyped for the 21 SNP (M1-M14 and 7 SNP related to $C Y P 11 A 1)$ and for two microsatellites (located 5' of CYP11A1 and SW1418). With the exception of M12, M13 and M14, all the markers were homozygous (data not shown). This female appeared to be homozygous for a large region (M1-M11) including SNP H3GA0021967. We examined the haplotypes of $480 \mathrm{LW}$ animals for 14 SNP (M1 to M14) from the CYP11A1 region and found that 14 animals shared the same two SSC7 chromosomal regions present in sow 65472. Nevertheless, genotyping for three markers inside the CYP11A1 gene did not detect any animal carrying the 65472 sow's haplotype. Thus, we believe that the number of genotyped SNP is sufficient to assume that sow 65472 is homozygous for a large region (M1-M11) including SNP H3GA0021967.

Since sow 65472 is considered as heterozygous for CYP11A1 expression level and homozygous for a haplotype in the M1-M11 large region, we hypothesized that the mutation controlling CYP11A1 expression is located downstream M11. This region was superimposed on the androstenone QTL region, which enabled us to suggest that a unique mutation located near the centre of the QTL region (M11-M14) could control androstenone accumulation in fat by regulating the CYP11A1 expression.

Table 1 Testis CYP11A1 expression in two LW families

\begin{tabular}{|c|c|c|c|c|c|}
\hline & \multirow[b]{2}{*}{ Haplotypes SSC7/SSC7 } & \multicolumn{3}{|c|}{ qPCR on RT products of RNA from testis } & \multirow{2}{*}{$\begin{array}{c}\text { [CYP11A1] } \\
\text { qPCR Arbitrary units }\end{array}$} \\
\hline & & & Descendant & Haplotypes SSC7/SSC7 & \\
\hline Boar 1 Pat1/Pat2 & Family A & Dam 61043 & 75082 & Mat1/Pat2 & 463 \\
\hline Boar 1 Pat1/Pat2 & Family A & Mat1/Mat2 & 75083 & Pat2/Mat1 & 224 \\
\hline Boar 1 Pat1/Pat2 & Family A & & 75084 & Pat1/Mat2 & 586 \\
\hline Boar 1 Pat1/Pat2 & Family A & & 75085 & Pat1/Mat2 & 199 \\
\hline Boar 1 Pat1/Pat2 & Family A & & 75086 & Pat1/Mat1 & 260 \\
\hline Boar 1 Pat1/Pat2 & Family A & & 75087 & Pat2/Mat2-Mat1 & 604 \\
\hline Boar 1 Pat1/Pat2 & Family A & & 75088 & Pat2/Mat2 & 408 \\
\hline Boar 1 Pat1/Pat2 & Family B & Dam 65472 & 75010 & Pat1/Mat3 & 1440 \\
\hline Boar 1 Pat1/Pat2 & Family B & Mat1/Mat3 & 75011 & Pat2/Mat3 & 1410 \\
\hline Boar 1 Pat1/Pat2 & Family B & & 75012 & Pat2/Mat1 & 273 \\
\hline Boar 1 Pat1/Pat2 & Family B & & 75014 & Pat2/Mat1 & 227 \\
\hline
\end{tabular}

In this table we report the results of the quantification of CYP11A1 expression in the testis [Cyp11A1]; contrary to animals in family A, in family B, the level of CYP11A1expression in descendants could be distinguished as a function of their maternal SSC7 chromosome; for more details on the characterisation of SSC7, [see additional file 1, Table S1] and for the quantification of CYP11A1 expression on testes for all the animals of the six families see Table 3. 
Table 2 Effects of various haplotypes of the M11-M14 region on Androstenone accumulation (480 LW)

\begin{tabular}{|c|c|c|c|c|c|c|c|}
\hline \multicolumn{3}{|c|}{$1^{\text {rst }}$ group of animals with haplotypes } & \multirow[b]{2}{*}{$P$} & \multicolumn{3}{|c|}{$2^{\text {nd }}$ group of animals with haplotypes } & \multirow[b]{2}{*}{ Effect on [andro] } \\
\hline nb animals & Haplotype SSC7 & [andro] & & $\mathrm{nb}$ animals & Haplotype SSC7 & [andro] & \\
\hline 303 & $\mathrm{GGAG} / \mathrm{X}$ or $\mathrm{GGAG} / \mathrm{GGAG}$ & $-0.11+/-0.63$ & 4.09E-07 & 116 & $x / X$ & $0.26+/-0.67$ & $\mathrm{GGAG}=$ andro - \\
\hline 102 & GGAG/GGAG & $-0.25+/-0.55$ & 0.00237 & 75 & GGAG/TAGA & $0.04+/-0.68$ & TAGA $=$ andro + \\
\hline 102 & GGAG/GGAG & $-0.25+/-0.55$ & 0.000822 & 22 & TAGG/TAGG & $0.36+/-0.71$ & TAGG $=$ andro + \\
\hline
\end{tabular}

Analysis of the M11, M12, M13 and M14 SNP set revealed five haplotypes (TAGA, TAGG, TGAG, GGAA and X for haplotypes other than these four) in the 480 animals of the LW population used for QTL mapping; [andro] is the mean $+/-$ SD of androstenone level in fat after transformation in log; $P=$ Student's $t$-test.

\section{Haplotype analysis in the centre of the QTL region}

We examined the haplotypes between M11-M14 in 480 LW animals (Table 2). The GGAG haplotype in the third group of SNP (M11-M12-M13-M14) occurred at a high frequency in the population $(0.48)$ and had a negative effect on androstenone level [10]. Since homozygous GGAG animals had a statistically significant lower level of androstenone than animals GGAG/TAGA or TAGG/ TAGG (Table 2), we suggest that haplotypes TAGA and TAGG could have a positive effect on androstenone level.

Furthermore, we analysed CYP11A1 expression in LW families according to haplotypes specifically of the QTL region. Haplotypes of the region between markers M11 and M14 in 22 animals characterised for CYP11A1 expression are shown in Table 3. Only two animals (75010 and 75011) considered as heterologous for CYP11A1 expression level had haplotype TGAG, the fourth haplotype characterised in the $480 \mathrm{LW}$ population and for which it was not possible to evaluate its effect on androstenone accumulation. Nevertheless these two animals have a paternal haplotype GGAG or TAGG which could have a contrary effect on androstenone level. Moreover, we found six animals GGAG/TAGA expected as $+/$ - and seven animals TAGG/TAGA expected as $+/+$ for androstenone accumulation with no significant difference in CYP11A1 expression (Table 3). Haplotypes of the

Table 3 Haplotypes of the M11-M14 region in 22 LW animals from six families

\begin{tabular}{|c|c|c|c|c|c|c|c|c|c|}
\hline \multirow[b]{3}{*}{ Boar } & \multirow[b]{3}{*}{ Sire haplotype } & \multirow[b]{3}{*}{ family } & \multirow[b]{3}{*}{ Sow } & \multirow[b]{3}{*}{ Animal } & \multicolumn{3}{|c|}{ Androstenone QTL } & \multirow{2}{*}{\multicolumn{2}{|c|}{$\begin{array}{c}\text { [CYP11A1] } \\
\text { real time PCR }\end{array}$}} \\
\hline & & & & & \multicolumn{2}{|c|}{ haplotypes } & \multirow[b]{2}{*}{ Expected (andro) } & & \\
\hline & & & & & Paternal allele & Maternal allele & & Individual value & Mean \\
\hline boar 1 & TAGG/GGAG & $A$ & 61043 & 75082 & TAGG & TAGA & $+/+$ & 463 & $361+/-463$ \\
\hline boar 1 & TAGG/GGAG & A & 61043 & 75083 & TAGG & TAGA & $+/+$ & 224 & $361+/-463$ \\
\hline boar 1 & TAGG/GGAG & A & 61043 & 75087 & TAGG & TAGA & $+/+$ & 605 & $361+/-463$ \\
\hline boar 1 & TAGG/GGAG & A & 61043 & 75088 & TAGG & TAGA & $+/+$ & 408 & $361+/-463$ \\
\hline boar 1 & TAGG/GGAG & B & 65472 & 75012 & TAGG & TAGA & $+/+$ & 273 & $361+/-463$ \\
\hline boar 1 & TAGG/GGAG & B & 65472 & 75014 & TAGG & TAGA & $+/+$ & 227 & $361+/-463$ \\
\hline boar 1 & TAGG/GGAG & & 69974 & 74999 & TAGG & TAGA & $+/+$ & 328 & $361+/-463$ \\
\hline boar 2 & GGAG/gggg & & 65477 & 75062 & GGAG & TAGA & $-/+$ & 220 & $368+/-236$ \\
\hline boar 1 & GGAG/gggg & & 65477 & 75063 & GGAG & TAGA & $-/+$ & 202 & $368+/-236$ \\
\hline boar 1 & TAGG/GGAG & A & 61043 & 75084 & GGAG & TAGA & $-/+$ & 586 & $368+/-236$ \\
\hline boar 1 & TAGG/GGAG & A & 61043 & 75085 & GGAG & TAGA & $-/+$ & 198 & $368+/-236$ \\
\hline boar 1 & TAGG/GGAG & A & 61043 & 75086 & GGAG & TAGA & $-/+$ & 260 & $368+/-236$ \\
\hline boar 1 & TAGG/GGAG & & 65529 & 75037 & GGAG & TAGA & $-/+$ & 743 & $368+/-236$ \\
\hline boar 2 & GGAG/gggg & & 65973 & 75108 & GGAG & GGAG & $-/-$ & 575 & \\
\hline boar 2 & GGAG/gggg & & 65973 & 75109 & GGAG & GGAG & $-/$ & 272 & \\
\hline boar 2 & GGAG/gggg & & 65973 & 75110 & GGAG & GGAG & $-/-$ & 299 & \\
\hline boar A & TAGG/GGAG & & 69974 & 74998 & GGAG & TAGG & $-/+$ & 276 & \\
\hline boar 2 & GGAG/gggg & & 65477 & 75061 & GGAG & TAGG & $-/+$ & 712 & \\
\hline boar 2 & GGAG/gggg & & 65973 & 75107 & gggg & TAGA & $? /+$ & 190 & \\
\hline boar 1 & TAGG/GGAG & & 65529 & 75036 & TAGG & TAGG & $+/+$ & 540 & \\
\hline boar 1 & TAGG/GGAG & B & 65472 & 75011 & TAGG & TGAG & $+/ ?$ & 1410 & \\
\hline boar 1 & TAGG/GGAG & B & 65472 & 75010 & GGAG & TGAG & $-/ ?$ & 1440 & \\
\hline
\end{tabular}

Haplotypes of the group of markers M11 to M14 were determined on 22 animals from six families; haplotype "gggg" in small letters is a haplotype not previously identified in the $480 \mathrm{LW}$ population; (andro) = expected androstenone level; [CYP11A1] = quantification of $\mathrm{CYP} 11 \mathrm{~A} 1$ expression in the testis. 
M11-M14 region in these $22 \mathrm{LW}$ animals characterised for CYP11A1 expression level are not concordant with those of the $480 \mathrm{LW}$ animals. Effects of the QTL region's haplotype on CYP11A1 expression level and androstenone accumulation are different.

\section{Conclusion}

This study suggests that the variation of CYP11A1 expression level is probably not regulated by a mutation located inside the CYP11A1 gene but rather by a mutation located downstream of the SNP H3GA0021967. In the French Large White population, the QTL for androstenone is mapped near this SNP. This co-location is probably a coincidence since haplotypes of the M11M14 region of animals characterised for CYP11A1 expression and of animals characterised for the QTL for androstenone are not concordant. This study shows that the testicular expression of CYP11A1 is not the main cause of this QTL for androstenone. As a conclusion, we propose to refute the hypothesis that a single mutation located near the centre of the QTL region (M11M14) could control androstenone accumulation in fat by regulating the CYP11A1 expression.

\section{Additional material}

Additional file 1: Presentation of genotypes of animals from the two families evaluated for the CYP11A1 expression. The data provide genotypes of eight markers allowing the characterisation of SSC7 haplotypes in a large region around CYP11A1 of animals from the two main LW families evaluated for CYP11A1 expression (families A and B)

\section{Acknowledgements}

We would especially like to thank the management and staff of Soviba for giving us access to the Saint-Maixent slaughterhouse and for their assistance in collecting samples. We would also like to thank the team running the genomic platform of the Génopole Toulouse Midi-Pyrénées (http://genopole-toulouse. prd.fr/index.php?lang=fr) for their contribution to data collection. The expression study was funded by the AVAMIP (Agence de Valorisation de la Région Midi-Pyrénées) through the MipAndro7 project. The genotyping of French LW was financed by the EC-funded FP6 Project "SABRE" (WP9).

\section{Author details}

'INRA, UMR444, Laboratoire de Génétique Cellulaire, 31326 Castanet-Tolosan, France. ${ }^{2}$ INRA, UMR1313, Génétique Animale et Biologie Intégrative (GABI), 78352 Jouy-en-Josas, France.

\section{Authors' contributions}

$A R$ and KF performed real-time PCR, sequencing, and data processing. $A R$ made the main contributions to the data analysis, data interpretation and drafting of the manuscript. GLM contributed very significantly to data interpretation. $\mathrm{CL}$ and JR supervised the experimental design and contributed to data interpretation and manuscript evaluation. Genotyping data acquisition was supervised by CL. All authors read and approved the final manuscript.

\section{Competing interests}

The authors declare that they have no competing interests.
Received: 15 February 2011 Accepted: 19 April 2011

Published: 19 April 2011

\section{References}

1. Zamaratskaia G, Squires EJ: Biochemical, nutritional and genetic effects on boar taint in entire male pigs. Animal 2009, 3:1508-1521.

2. Robic A, Larzul C, Bonneau M: Genetic and metabolic aspects of androstenone and skatole deposition in pig adipose tissue: A review. Genet Sel Evol 2008, 40:129-143.

3. Katkov T, Gower DB: The biosynthesis of androst-16-enes in boar testis tissue. Biochem J 1970, 117:533-538.

4. Kwan T, Orengo C, Gower DB: Biosynthesis of androgens and pheromonal steroids in neonatal porcine testicular preparations. FEBS letters 1985, 183:359-364.

5. Gower DB, 16-unsaturated C19 steroids: A review of their chemistry, biochemistry and possible physiological role. J Steroid Biochem 1972, 3:45-103.

6. Duijvesteijn N, Knol EF, Merks JW, Crooijmans RP, Groenen MA, Bovenhuis $\mathrm{H}$, Harlizius B: A genome-wide association study on androstenone levels in pigs reveals a cluster of candidate genes on chromosome 6. BMC Genet 2010, 11:42.

7. Lee GJ, Archibald AL, Law AS, Lloyd S, Wood J, Haley CS: Detection of quantitative trait loci for androstenone, skatole and boar taint in a cross between Large White and Meishan pigs. Anim Genet 2005, 36:14-22.

8. Quintanilla R, Demeure O, Bidanel JP, Milan D, lannuccelli N, Amigues Y, Gruand J, Renard C, Chevalet C, Bonneau M: Detection of quantitative trait loci for fat androstenone levels in pigs. J Anim Sci 2003, 81:385-394.

9. Grindflek E, Meuwissen THE, Aasmundstad T, Hamland H, Hansen MHS, Nome T, Kent M, Torjesen P, Lien S: Revealing genetic relationships between compounds affecting boar taint and reproduction in pigs. J Anim Sci 2011, 89:680-692.

10. Le Mignon G, lannuccelli N, Robic A, Billon Y, Bidanel JP, Larzul C: Fine mapping of quantitative trait loci for androstenone and skatole levels in pig. Proceedings of the 9th World Congress on Genetics Applied to Livestock Production: 1-6 August 2010; Leipzig 2010 [http://www.kongressband.de/ wcgalp2010/assets/pdf/0212.pdf].

11. Moe M, Meuwissen T, Lien S, Bendixen C, Wang X, Conley LN, Berget I, Tajet H, Grindflek E: Gene expression profiles in testis of pigs with extreme high and low levels of androstenone. BMC Genomics 2007, 8:405.

12. Grindflek E, Berget I, Moe M, Oeth P, Lien S: Transcript profiling of candidate genes in testis of pigs exhibiting large differences in androstenone levels. BMC Genet 2010, 11:4.

13. Greger DL, Antle S, Plath-Gabler A, Gutshall K, Mills E, Hammerstedt R, Hagen D, Baumrucker C: Identification of a porcine CYP11A1 polymorphism and associations with steroid concentrations and reproductive traits in pigs. Plant and Animal Genome IX Conference: 13-17 January 2001; San Diego 2001 [http://www.intl-pag.org/9/abstracts/P5m_05. html].

14. Miller WL: Mitochondrial specificity of the early steps in steroidogenesis. J Steroid Biochem Mol Biol 1995, 55:607-616.

15. Archibald A, Bolund L, Churcher C, Fredholm M, Groenen MA, Harlizius B, Lee KT, Milan D, Rogers J, Rothschild MF, Uenishi H, Wang J, Schook LB: Pig genome sequence - analysis and publication strategy. BMC Genomics 2010, 11:438.

16. Courcelle E, Beausse Y, Letort S, Stahl O, Fremez R, Ngom-Bru C, Gouzy J, Faraut T: Narcisse: a mirror view of conserved syntenies. Nucleic Acids Res 2008, 36:D485-490.

17. Greger D: Genetic marker for meat quality, growth, carcass and reproductive traits in livestock. Patent WO 0/69882 Washington, DC; 2000

doi:10.1186/1297-9686-43-15

Cite this article as: Robic et al: New investigations around CYP11A1 and its possible involvement in an androstenone QTL characterised in Large White pigs. Genetics Selection Evolution 2011 43:15. 